\title{
Similarities and differences between multiple inflammatory syndrome in children associated with COVID-19 and Kawasaki disease: clinical presentations, diagnosis, and treatment
}

\author{
Qing-You Zhang ${ }^{1} \cdot$ Bo-Wen $\mathrm{Xu}^{1} \cdot$ Jun-Bao $\mathrm{Du}^{1}$
}

Received: 7 January 2021 / Accepted: 29 April 2021 / Published online: 20 May 2021

(c) Children's Hospital, Zhejiang University School of Medicine 2021

\begin{abstract}
Background Severe acute respiratory syndrome coronavirus 2 (SARS-CoV-2) infection has been spreading rapidly around the world, while "multisystem inflammatory syndrome in children" (MIS-C) is a new type of syndrome that has now been reported in many countries. Similar and different characteristics between KD and MIS-C have been reported in a variety of literature. We aimed to focus on reviewing clinical presentations, diagnosis, and treatment of KD and MIS-C.

Methods We searched articles in the electronic databases, including the Cochrane Library database, EMBASE, and MEDLINE with the keywords "multiple inflammatory syndrome" and/or "COVID-19" and/or "Kawasaki disease" and "children". Results Main presentations of MIS-C and KD include fever, rashes, mucous membrane involvement, conjunctivitis, hands and feet erythema/edema, and cervical lymphadenopathy. However, compared with the highest incidence of KD among some Asian countries, MIS-C is common among Black and Hispanic children. MIS-C is common in older children and teenagers, whereas classic $\mathrm{KD}$ is common in children under five years of age. Gastrointestinal symptoms, shock, and coagulopathy are common in MIS-C patients but are not common in classic KD. Cardiac manifestations are more common than KD, including myocarditis with cardiac dysfunction and coronary artery dilation or aneurysms. Severe cases in MIS-C present with vasodilated or cardiogenic shock that requires fluid resuscitation, muscular support, and even mechanical ventilation and extracorporeal membrane oxygenation (ECMO), whereas $\mathrm{KD}$ rarely presents with these manifestations and requires these treatments. Increased serum ferritin, leukopenia, lymphopenia and thrombocytopenia are common in MIS-C. However, thrombocytosis is a characteristic feature of KD. Intravenous immunoglobulin (IVIG) and moderate-high dose aspirin are still a standard recommended treatment for KD. In addition to the above-mentioned medications, steroids and biological drugs are frequently used in patients with MIS-C. Most of the children with KD have a good prognosis; however, the longterm clinical outcomes of MIS-C are not clear.

Conclusions The overall presentation and treatment of MIS-C appear to overlap with KD. However, there are still great differences between the syndromes, and it is controversial to say whether MIS-C is a new entity or is a "severe type" of KD.
\end{abstract}

Keywords Kawasaki disease $\cdot$ Multisystem inflammatory syndrome $\cdot$ Children

\section{Introduction}

A new syndrome in children and juveniles was first described in the global pandemic of coronavirus disease 2019 (COVID-19), termed as "multisystem inflammatory

Jun-Bao Du

junbaodu1@126.com

1 Department of Pediatrics, Peking University First Hospital, Xi-An Men Street No. 1, West District, Beijing 100034, China syndrome in children" (MIS-C), which is closely associated with COVID-19 infection [1-3]. So far, a series of cases with such a syndrome have been reported in children from Italy [3], the UK [4], Spain [5], France [6, 7], the United States [8], India [9], Brazil [10] and many other countries including South Korea [11]. No reports of MIS-C have been observed in China and Singapore. This syndrome has clinical manifestations similar to Kawasaki disease (KD) and Kawasaki disease shock syndrome (KDSS) [12], or KD with macrophage activation syndrome (MAS) [13]. Patients may present with high fever, lymphadenopathy, pleomorphic rash, conjunctivitis, mucosal pathology changes, and 
coronary artery dilation. Severe cases may present in cardiovascular shock, encephalitis, and multiple organ failure [8, 14-16]. However, obvious differences have also been found between the two syndromes in clinical presentations, diagnosis, and treatment.

The differences between MIS-C and KD might be associated with different pathogenesis of the two diseases. Unfortunately, the pathogenesis of two diseases is still under research and is not yet clear. There are differences between MIS-C and KD in hyper-inflammatory states. For example, interleukin (IL)-17A, the main contributor in the cytokine storm, is significantly higher in KD than that in MIS-C. Apart from IL-17A, a plasma protein called endothelial and smooth muscle cell-derived neuropilin-like protein (ESDN) was obviously elevated in KD as compared with that in MIS$\mathrm{C}$, which is related to arterial damage [17]. Other studies have reported that interferon- $\gamma$ (IFN- $\gamma$ )-associated cytokines, IL-1 associated cytokines, and inflammatory monocytes are major contributors to acute inflammation in MIS-C and KD patients, and most of these studies overlap. However, compared with KD patients, a subgroup of MIS-C patients showed a significant increase in circulating levels of IFN- $\gamma$ which is the hallmark of MAS [18]. These studies suggest that immune-modulating factors for MIS-C and KD may contribute differently to each condition.

In this review, we discussed the similarities and differences between MIS-C associated with COVID-19 and KD based on clinical manifestations, diagnosis, and treatment (Fig. 1).

\section{Clinical presentations of MIS-C and KD: similarities and differences}

Although the first case of MIS-C was reported in early April 2020 [19], the real concern was raised on April 26, 2020. On that day, National Health Service in the United Kingdom (NHS, UK) issued a warning that severely ill children may have similar features as that of the toxic shock syndrome, severe COVID-19 infection, and atypical KD [20]. On May 14, 2020, the Center for Disease Control and Prevention (CDC) issued a health advisory to report the cases that matched the criteria for MIS-C [21], which was also emphasized by others, including the Royal College of Paediatrics and Child Health (RCPCH), the World Health Organization (WHO), and the US Center for Disease Control and Prevention (CDC) [22]. All three institutions developed their definitions, namely, pediatric inflammatory multisystem syndrome temporally associated with SARS-CoV-2 (PIMS-TS), MIS-C by CDC, US and the WHO [21, 22]. All these definitions include the age of the disease onset, clinical characteristics, laboratory examination of inflammation, and laboratory or epidemiologic proof of SARS-CoV-2 infection. However, there are other excluded conditions, such as toxic shock syndrome, hemophagocytic lymphohistiocytosis, etc. As per the definition of MIS-C by CDC of the United States [22], there are six criteria as follows: the illness is severe enough to require hospitalization; patients are below 21 years; the body temperature of patients is over $38.0{ }^{\circ} \mathrm{C}$ or self-conscious fever that lasts for at least $24 \mathrm{~h}$; inflammation found through

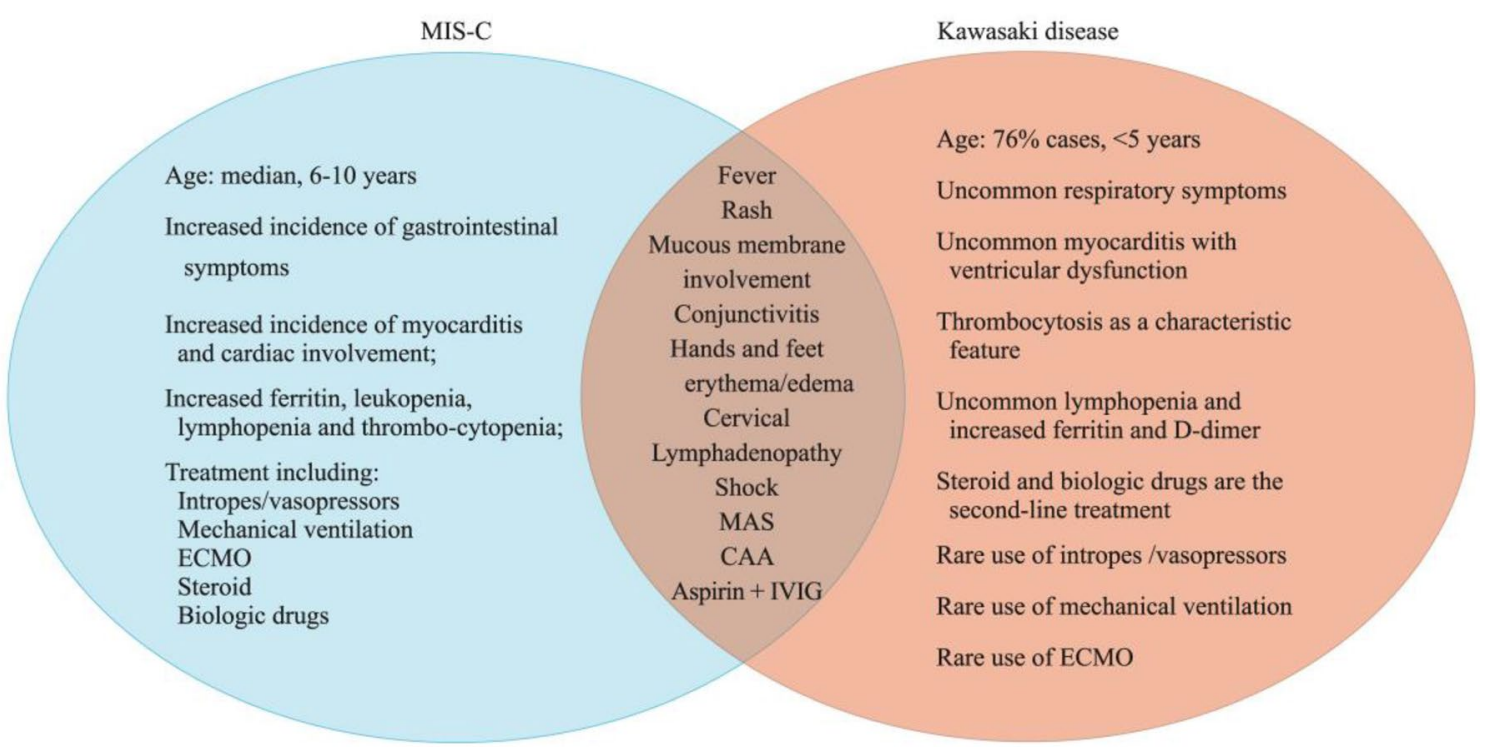

Fig. 1 Similarities and differences between multiple inflammatory syndrome in children (MIS-C) associated with COVID-19 and Kawasaki disease (KD): the clinical presentations, diagnosis, and treatment. ECMO extracorporeal membrane oxygenation, $M A S$ macrophagic activation syndrome, $C A A$ coronary artery aneurysm, IVIG intravenous immunoglobulin 
a laboratory clue; at least two organ systems showing signs of involvement; laboratory evidence that confirms SARS$\mathrm{CoV}-2$ infection (SARS-CoV-2 DNA being positive for real-time reverse-transcriptase polymerase chain reaction, RT-PCR or the presence of a positive antibody) or a positive epidemiological history. However, SARS-CoV-2 infection is not an essential factor in the UK guideline.

Data on the characteristics of children and adolescents with MIS-C remain limited and are updated daily. The median age of the affected children in MIS-C ranges from 6 to 10 years, which is different from the classical KD because MIS-C affects older children and teenagers [23]. 76\% of the cases of KD are under 5 years old [24]. However, the older patients of KD are known to be more severe than younger patients. Also, MIS-C seems to be more common in males than in females. A majority of papers have reported that more than $60 \%$ of patients were found to be males [2-10, 23]. MIS-C and KD are similar in many clinical manifestations; for example, both exhibits persistent high fever, $25-65.5 \%$ of the patients with symptoms of MIS-C are also consistent with the classic or incomplete $\mathrm{KD}$, including rashes, mucous membrane involvement, conjunctivitis, hands and feet erythema/edema, and cervical lymphadenopathy [2-10]. However, the differences in clinical manifestations between the two diseases are also obvious. Gastrointestinal symptoms, such as vomiting, abdominal pain, and/ or diarrhea, are more pronounced in children with MIS-C than the ones with KD [2-10, 23, 25, 26]. According to the report of Abdel-Haqet et al. the abdominal imaging findings of their MIS-C patients were lymphadenopathy, hepatosplenomegaly, mesenteric adenitis, calculous cholecystitis, pancreatitis, ascites, intestinal wall thickening, and inflammation [26]. Because some KD cases did not have abdominal ultrasound examination, the abovementioned gastrointestinal findings seem not common in KD [27]. Also, the patients with MIS-C are more serious than the children with KD. $33-87 \%$ of MIS-C patients experienced hypotension that required inotropic support. However, only $2-7 \%$ of children with KD developed shock [2-10, 21, 22, 25, 28]. According to previous reports, $1.1-1.9 \%$ of children with KD could be associated with macrophage activation syndrome (MAS) $[28,29]$. However, MAS is more frequently seen in children with MIS-C, with an incidence of 3-50\%. MAS can lead to multi-organ system dysfunction and is associated with high morbidity and mortality $[2,13,14]$. Respiratory distress is rarely seen in KD patients, whereas, in MIS-C, $12-46 \%$ of the patients receive oxygen or mechanical ventilation [2-10].

Cardiac manifestations of these two diseases are also different, although both can have myocarditis and coronary artery dilation and aneurysm (CAA). After being treated with intravenous immunoglobulin (IVIG), CAA can be seen in only about $4 \%$ of the patients with KD [28], while in MIS-C, $14-36 \%$ of patients exhibit CAA, which is higher than those seen in patients with $\operatorname{KD}[15,16]$. Some researchers found that CAA observed in patients with MIS-C is generally reversible [16]. Also, the left ventricular systolic dysfunction (LV ejection fraction $<50 \%$ ) is rarely seen in KD, but in MIS-C, $18-87 \%$ of children display ventricular dysfunction [15, 16, 23] (Table 1). Nevertheless, the long-term prognosis of CAA caused by MIS-C is unclear.

\section{Laboratory features of MIS-C and KD: similarities and differences}

Elevated inflammatory indexes occur in both MIS-C and $\mathrm{KD}$ cases. However, the elevation observed in KD cases was moderate [28] compared to the MIS-C, where an obvious increase was observed in the levels of CRP, PCT, and IL-6 [2-10, 23, 25], with the values being more than 350 times, 16 times, and 3 times higher, respectively [25]. Lymphopenia was present in $37-81 \%$ of MIS-C patients [2-10, 23], whereas in patients with KD, it was rare. However, thrombocytopenia and the ratio of neutrophils are common risk factors of refractory KD in the acute phase [28]. In addition, the laboratory characteristics were different according to age [30]. Thrombocytosis is a characteristic feature of the convalescent KD. The majority of patients with MIS-C presented with thrombocytopenia $<150 \times 10^{9} / \mathrm{L}$ [2-10]. Another characteristic of patients with MIS-C is a marked increase in the biochemical markers of cardiac injury, including troponin $\mathrm{T}$ and brain natriuretic peptide (BNP) [2-10, 15, 16, 23], whereas these indexes increase only slightly in children with $\mathrm{KD}$, which indicates that children with MIS-C may have more serious cardiac involvement $[15,16]$. Highly elevated ferritin and D-dimer were widely reported in children with MISC, which was also similar to that of the MAS [2-10, 23, 25]. These findings are uncommon in classic KD and may help to differentiate these two diseases. However, some researchers reported that risk factors for coronary artery disease in patients with KD include elevated D-dimer levels $[31,32]$. The most characteristic laboratory test of MIS-C is the one that confirms the SARS-CoV-2 infection, including a positive SARS-CoV-2 DNA RT-PCR or a positive antibody test. Most patients with MIS-C (75-100\%) have IgG antibodies for SARS-CoV-2, but only a few tested positive for RT-PCR with nasopharyngeal and oropharyngeal swab sampling [2-10, 23]. These results suggest that MIS-C is not the result of acute viral infection, but the virus is the inducing agent for the uncontrolled immune response in susceptible children [17, 33]. Compared to the peak of SARS-CoV-2 infection, the cases of MIS-C appeared late in some countries. This clinical phenomenon also supports the above hypothesis [34] (Table 1). 
Table 1 Differences of multiple inflammatory syndrome in children associated with COVID-19 and Kawasaki disease

\begin{tabular}{|c|c|c|}
\hline Items & MIS-C & $\mathrm{KD}$ \\
\hline Age & Median age: $6-10$ years & $76 \%$ cases $<5$ years \\
\hline Sex & More common in male & Male: female about $1.5: 1$ \\
\hline Ethnic distribution & $\begin{array}{l}\text { High proportion of African ethnicity or ancestry, as } \\
\text { well as Hispanic subjects }\end{array}$ & $\begin{array}{l}\text { Asian children have the } \\
\text { highest incidence in the } \\
\text { world }\end{array}$ \\
\hline \multicolumn{3}{|l|}{ Clinical features } \\
\hline Fever & $100 \%$ & $100 \%$ \\
\hline Rash & $50-75 \%$ & Above $90 \%$ \\
\hline Mucous membrane involvement & $60-94 \%$ & Above $90 \%$ \\
\hline Conjunctivitis & $48-69 \%$ & Above $90 \%$ \\
\hline Hands and feet erythema/edema & $26-68 \%$ & About $75 \%$ \\
\hline Cervical lymphadenopathy & $30-70 \%$ & $50-70 \%$ \\
\hline Classic and incomplete KD presentation & $25-65.5 \%$ & $100 \%$ \\
\hline Shock (KDSS) & $33-87 \%$ & $2-7 \%$ \\
\hline MAS & $3-50 \%$ & $1.1-1.9 \%$ \\
\hline \multicolumn{3}{|l|}{ Other clinical features } \\
\hline Gastrointestinal symptoms & $60-100 \%$ & Rare \\
\hline Respiratory distress & $12-46 \%$ & Rare \\
\hline Cardiac manifestation & $18-87 \%$ & Rare \\
\hline CAA & $14-36 \%$ & $4 \%$ \\
\hline \multicolumn{3}{|l|}{ Laboratory features } \\
\hline Lymphopenia & $37-81 \%$ & Rare \\
\hline Platelets & Decreased & Increased \\
\hline Level of inflammatory markers & Noticeably increased & Increased \\
\hline Troponin $\mathrm{T}$ & More common & Rare \\
\hline BNP & Marked increased & Mild increased \\
\hline Ferritin & Highly elevated & Normal \\
\hline D-dimer & Significantly increase & Normal \\
\hline SARS-Cov2 positive (serology or PCR) & $80-100 \%$ & None \\
\hline \multicolumn{3}{|l|}{ Treatment } \\
\hline Intropes/vasopressors & $25-100 \%$ & Rare \\
\hline Mechanical ventilation & $13-57 \%$ & Rare \\
\hline Aspirin & $3-28 \%$ & Very rare \\
\hline ECMO & In some cases $(0 \%-75 \%)$ & $100 \%$ \\
\hline IVIG & $54-100 \%$ & $100 \%$ \\
\hline Steroid & $20-83 \%$ & In some cases \\
\hline Biologic drugs & $7-80 \%$ & Occasionally reported \\
\hline \multicolumn{3}{|l|}{ Outcomes } \\
\hline Mortality & About $2.1-18 \%$ & About $0.17 \%$ \\
\hline
\end{tabular}

$M I S-C$ multiple inflammatory syndrome in children, $M A S$ macrophagic activation syndrome, $C A A$ coronary artery aneurysm, $I V I G$ intravenous immunoglobulin, $K D$ Kawasaki disease, $P C R$ polymerase chain reaction, ECMO extracorporeal membrane oxygenation, KDSS Kawasaki disease shock syndrome, $B N P$ brain natriuretic peptide

\section{Treatment of MIS-C and KD: similarities and differences}

The standard recommended treatment for KD is a high dose of intravenous immunoglobulin (IVIG), i.e., $2 \mathrm{~g} / \mathrm{kg}$ as a single infusion, along with a moderately high dose of acetylsalicylic acid (ASA). The infusion time is recommended to be over $10-12 \mathrm{~h}$ [26]. Nearly $10-20 \%$ of the KD cases are refractory. Generally, fever develops or persists for $36 \mathrm{~h}$ after IVIG infusion. Administration of the second dose of IVIG, high-dose pulse steroids, or infliximab may be considered in patients having refractory KD as per the AHA guidelines [28]. However, current treatment strategies of MIS-C are empirical and do not have enough evidence. 
Treatment strategies include symptomatic and supportive therapy, along with immunomodulatory treatment. Because the patients with MIS-C were more serious than those with $\mathrm{KD}$, most of them were hospitalized in the intensive care unit (ICU), where $25-100 \%$ needed inotropic support or vasopressors [2-10, 23, 25]. Currently, it is suggested that immune-mediated damage triggered by SARS-CoV-2 infection, other than the virus itself, leads to MIS-C. $13-57 \%$ of patients with MIS-C receive invasive mechanical ventilator support. Also, ECMO support was used in 3-28\% of patients with MIS-C [2-10]. However, all the above-mentioned life support therapies were rarely required in patients with KD. Because children with MIS-C showed KD-like symptoms, a majority (ranging from 54 to $100 \%$ ) of them received IVIG. Also, the response to IVIG was worse in patients with MIS-C than with the KD. Most of the children with MIS-C (about 20-83\%) needed a second dose of IVIG or steroids [2-10, 15, 16, 23, 25]. Also, the efficacy of acetylsalicylic acid (ASA), the traditional drug used to treat KD, in MIS-C was uncertain. Some studies reported that $75 \%$ of the patients with MIS-C received ASA [2-10, 23]. It is rational to provide antiplatelet treatment along with a low dose of ASA for MIS-C patients who have evidence of coronary involvement [14-16]. For the patients with KD who are refractory to first-line of IVIG therapy, a combination or steroid-free treatment with biological drugs, such as infliximab, should be considered [28]. The release of excessive pro-inflammatory cytokines occurs in both MIS-C and $\mathrm{KD}$ cases, and the "cytokine storm" is found in adults with SARS-CoV-2, too. Frequently, about $7-80 \%$ of biological drugs are added as a supplemental therapy for MIS-C patients, especially for those with clinical features consistent with those of the MAS [2-10, 13, 14, 23, 35] (Table 1).

\section{Outcomes of MIS-C and KD: similarities and differences}

Most children with KD have a good prognosis, where the in-hospital mortality rate is about $0.17 \%$, and $0.015 \%$ in Japan [28]. The long-term clinical outcomes of MIS-C are not clear because it is a new syndrome that is relevant to the SARS-CoV-2 infection. The in-hospital mortality rate of MIS-C varied greatly with different centers. Although some studies reported no deaths in the series of cases with MIS-C, other studies reported a mortality rate of up to $18 \%$ [10] (Table 1).

\section{Conclusions}

A new syndrome in children known as MIS-C, related to the infection with SARS-CoV-2, has recently been reported in many countries and regions with a global COVID-19 pandemic. MIS-C shares many clinical features with KD. However, there are still great differences between both the syndromes, and it is controversial to say whether MIS-C is a new entity or a "severe type" of KD [35, 36]. The characteristics of COVID-19 infection in children and the possible mechanistic links between COVID-19 and KD require further studies from a global perspective.

However, at present, it is difficult to identify clearly between "severe type" of KD and MIS-C because the "severe type" KD is very rare in patients with KD. Only $2-7 \%$ of children with KD developed shock [28], and $1.1-1.9 \%$ of children are associated with MAS [29], which is the limitation of the present review. However, accumulated experiences in the management of severe cases of KD will make it probable to differentiate "severe type" of KD from MIS-C.

Author contributions QZ had primary responsiblity for the design and execution of the study, data collection, preliminary data analysis and writing the manuscript. BX participated in data collection, preliminary data analysis and the writing of the manuscript.JD supervised the design and execution of the study, performed the final data analyses and contributed to the writing of the manuscript.

Funding This research was supported by grants from the National Natural Science Foundation of China (7171010).

\section{Compliance with ethical standards}

Ethical approval The ethics committee of Peking University First Hospital granted an exemption from requiring ethics approval.

Conflict of interest Professor Junbao Du serves as an associate editor for this journal. The paper was handled by the other Editor and has undergone rigrous peer review process. None of the authors participated in the journal's review of, or decisions related to this manuscript. All authors declared no finanacial or non-financial conflict of interests related to this paper.

\section{References}

1. Lu X, Zhang L, Du H, Zhang J, Li YY, Qu J, et al. SARS-CoV-2 infection in children. N Engl J Med. 2020;382:1663-5.

2. Riphagen S, Gomez X, Gonzalez-Martinez C, Wilkinson N, Theocharis P. Hyperinflammatory shock in children during COVID-19 pandemic. Lancet. 2020;395:1607-8.

3. Verdoni L, Mazza A, Gervasoni A, Martelli L, Ruggeri M, Ciuffreda M, et al. An outbreak of severe Kawasaki-like disease at the Italian epicentre of the SARS-CoV-2 epidemic: an observational cohort study. Lancet. 2020;395:1771-8.

4. Whittaker E, Bamford A, Kenny J, Kaforou M, Jones CE, Shah $\mathrm{P}$, et al. Clinical characteristics of 58 children with a pediatric inflammatory multisystem syndrome temporally associated with SARS-CoV-2. JAMA. 2020;324:259-69.

5. Moraleda C, Serna-Pascual M, Soriano-Arandes A, Simó S, Epalza C, Santos M, et al. Multi-inflammatory syndrome in children related to SARS-CoV-2 in Spain. Clin Infect Dis. 2021;72:e397-401. 
6. Toubiana J, Poirault C, Corsia A, Bajolle F, Fourgeaud J, Angoulvant $\mathrm{F}$, et al. Kawasaki-like multisystem inflammatory syndrome in children during the Covid-19 pandemic in Paris, France: prospective observational study. BMJ. 2020;369:m2094.

7. Belot A, Antona D, Renolleau S, Javouhey E, Hentgen V, Angoulvant $\mathrm{F}$, et al. SARS-CoV-2-related paediatric inflammatory multisystem syndrome, an epidemiological study, France, 1 March to 17 May 2020. Euro Surveill. 2020;25:2001010.

8. Feldstein LR, Rose EB, Horwitz SM, Collins JP, Newhams MM, Son MBF, et al. Multisystem inflammatory syndrome in U.S. children and adolescents. N Engl J Med. 2020;383:334-46.

9. Dhanalakshmi K, Venkataraman A, Balasubramanian S, Madhusudan M, Amperayani S, Putilibai S, et al. Epidemiological and clinical profile of pediatric inflammatory multisystem syndrome - temporally associated with SARS-CoV-2 (PIMS-TS) in Indian children. Indian Pediatr. 2020;57:1010-4.

10. Lima-Setta F, Magalhães-Barbosa MC, Rodrigues-Santos G, Figueiredo E, Jacques ML, Zeitel RS, et al. Multisystem inflammatory syndrome in children (MIS-C) during SARS-CoV-2 pandemic in Brazil: a multicenter, prospective cohort study. J Pediatr (Rio J). 2020;S0021-7557:30225-4.

11. Choe YJ, Choi EH, Choi JW, Eun BW, Eun LY, Kim YJ, et al. Surveillance of COVID-19-associated multisystem inflammatory syndrome in children, South Korea . Emerg Infect Dis. 2021;27:1196-200.

12. Koné-Paut I, Cimaz R. Is it Kawasaki shock syndrome, Kawasakilike disease or pediatric inflammatory multisystem disease? The importance of semantic in the era of COVID-19 pandemic. RMD Open. 2020;6:e001333.

13. Loomba RS, Villarreal EG, Flores S. COVID-19 and hyperinflammatory syndrome in children: Kawasaki disease with macrophage activation syndrome in disguise? Cureus. 2020;12:e9515.

14. Nakra NA, Blumberg DA, Herrera-Guerra A, Lakshminrusimha S. Multi-system inflammatory syndrome in children (MIS-C) following SARS-CoV-2 infection: review of clinical presentation, hypothetical pathogenesis, and proposed management. Children (Basel). 2020;7:69.

15. Sperotto F, Friedman KG, Son MBF, VanderPluym CJ, Newburger JW, Dionne A. Cardiac manifestations in SARS-CoV-2-associated multisystem inflammatory syndrome in children: a comprehensive review and proposed clinical approach. Eur J Pediatr. 2021;180:307-22.

16. Alsaied T, Tremoulet AH, Burns JC, Saidi A, Dionne A, Lang $\mathrm{SM}$, et al. Review of cardiac involvement in multisystem inflammatory syndrome in children. Circulation. 2021;143:78-88.

17. Consiglio CR, Cotugno N, Sardh F, Pou C, Amodio D, Rodriguez $\mathrm{L}$, et al. The immunology of multisystem inflammatory syndrome in children with COVID-19. Cell. 2020;183:968-81.e7.

18. Esteve-Sole A, Anton J, Pino-Ramirez RM, Sanchez-Manubens J, Fumadó V, Fortuny C, et al. Similarities and differences between the immunopathogenesis of COVID-19-related pediatric multisystem inflammatory syndrome and Kawasaki disease. J Clin Invest. 2021;131:e144554.

19. Jones VG, Mills M, Suarez D, Hogan CA, Yeh D, Segal JB, et al. COVID-19 and Kawasaki disease: Novel virus and novel case. Hosp Pediatr. 2020;10:537-40.

20. Rowley AH. Understanding SARS-CoV-2-related multisystem inflammatory syndrome in children. Nat Rev Immunol. 2020;20:453-4.

21. CDC Health Alert Network. Multisystem inflammatory syndrome in children (MIS-C) associated with Coronavirus disease 2019
(COVID-19). 2020. https://emergency.cdc.gov/han/2020/han00 432.asp. Accessed 23 May 2020.

22. World Health Organization. Multisystem inflammatory syndrome in children and adolescents temporally related to COVID- 19. 2020. https://www.who.int/news-room/commentaries/detail/ multisystem-inflammatory-syndrome-in-children-and-adole scents-with-covid-19.

23. Felsenstein S, Willis E, Lythgoe H, McCann L, Cleary A, Mahmood K, et al. Presentation, treatment response and shortterm outcomes in paediatric multisystem inflammatory syndrome temporally associated with SARS-CoV-2 (PIMS-TS). J Clin Med. 2020;9:3293.

24. Chang RK. The incidence of Kawasaki disease in the United States did not increase between 1988 and 1997. Pediatrics. 2003;111:1124-5.

25. Zou H, Lu J, Liu J, Wong JH, Cheng S, Li Q, et al. Characteristics of pediatric multi-system inflammatory syndrome (PMIS) associated with COVID-19: a meta-analysis and insights into pathogenesis. Int J Infect Dis. 2021;102:319-26.

26. Abdel-Haq N, Asmar BI, Deza Leon MP, McGrath EJ, Arora HS, Cashen K, et al. SARS-CoV-2-associated multisystem inflammatory syndrome in children: clinical manifestations and the role of infliximab treatment. Eur J Pediatr. 2021;180:1581-91.

27. Colomba C, La Placa S, Saporito L, Corsello G, Ciccia F, Medaglia A, et al. Intestinal involvement in Kawasaki disease. J Pediatr. 2018;202:186-93.

28. McCrindle BW, Rowley AH, Newburger JW, Burns JC, Bolger AF, Gewitz M, et al. Diagnosis, treatment, and long-term management of Kawasaki disease: A scientific statement for health professionals from the American Heart Association. Circulation. 2017; 135:e927-99.

29. Han SB, Lee SY. Macrophage activation syndrome in children with Kawasaki disease: diagnostic and therapeutic approaches. World J Pediatr. 2020;16:566-74.

30. Kwak JH, Lee J, Ha KS. Significance of differential characteristics in infantile Kawasaki disease. Korean Circ J. 2019;49:755-65.

31. Masuzawa Y, Mori M, Hara T, Inaba A, Oba MS, Yokota S. Elevated $\mathrm{D}$-dimer level is a risk factor for coronary artery lesions accompanying intravenous immunoglobulin-unresponsive Kawasaki disease. Ther Apher Dial. 2015;19:171-7.

32. Zhou Y, Wang S, Zhao J, Fang P. Correlations of complication with coronary arterial lesion with VEGF, PLT, D-dimer and inflammatory factor in child patients with Kawasaki disease. Eur Rev Med Pharmacol Sci. 2018;22:5121-6.

33. Frenkel LD, Gomez F, Bellanti JA. COVID-19 in children: Pathogenesis and current status. Allergy Asthma Proc. 2021;42:8-15.

34. Ouldali N, Pouletty M, Mariani P, Beyler C, Blachier A, Bonacorsi S, et al. Emergence of Kawasaki disease related to SARSCoV-2 infection in an epicentre of the French COVID-19 epidemic: a time-series analysis. Lancet Child Adolesc Health. 2020;4:662-8.

35. Xu S, Chen M, Weng J. COVID-19 and Kawasaki disease in children. Pharmacol Res. 2020;159:104951.

36. Loomba RS, Villarreal E, Flores S. COVID-19 and Kawasaki syndrome: should we really be surprised? Cardiol Young. 2020;30:1059-60.

Publisher's Note Springer Nature remains neutral with regard to jurisdictional claims in published maps and institutional affiliations. 